\title{
Clinical Trial Epoch
}

National Cancer Institute

\section{Source}

National Cancer Institute. Clinical Trial Epoch. NCI Thesaurus. Code C71738.

A portion of a study containing one or more study segments with a consistent objective such as screening subjects or treating disease. 